\title{
Global existence of solutions for a class of thermoelastic plate systems
}

Yang $\operatorname{Liu}^{1,2^{*}}$ (D)

\section{"Correspondence:}

liuyangnufn@163.com

'College of Mathematics and Computer Science, Northwest Minzu University, Lanzhou, People's Republic of China

${ }^{2}$ College of Mathematics, Sichuan University, Chengdu, People's

Republic of China

\begin{abstract}
This paper is concerned with the initial-boundary value problem for a class of thermoelastic plate systems. Under some appropriate assumptions, the global existence of solutions is obtained.
\end{abstract}

AMS Subject Classification: Primary 35A01; 35D30; $35 G 61$

Keywords: Thermoelastic plate systems; Initial-boundary value problem; Global existence

\section{Introduction}

In this paper, we study the following initial-boundary value problem for a class of thermoelastic plate systems:

$$
\left\{\begin{array}{l}
u_{t t}+\Delta^{2} u-g\left(\|\nabla u\|_{L^{2}(\Omega)}^{2}\right) \Delta u-\Delta u_{t t}+v \Delta \theta=f(u), \quad(x, t) \in \Omega \times \mathbb{R}^{+}, \\
\theta_{t}-\omega \Delta \theta-(1-\omega) \int_{0}^{\infty} k(\tau) \Delta \theta(t-\tau) \mathrm{d} \tau-v \Delta u_{t}=0, \quad(x, t) \in \Omega \times \mathbb{R}^{+},
\end{array}\right.
$$

with boundary conditions

$$
\left\{\begin{array}{l}
u=\Delta u=0, \quad(x, t) \in \partial \Omega \times[0, \infty) \\
\theta=0, \quad(x, t) \in \partial \Omega \times \mathbb{R}
\end{array}\right.
$$

and initial conditions

$$
\left\{\begin{array}{l}
u(x, 0)=u_{0}(x), \quad u_{t}(x, 0)=u_{1}(x), \quad x \in \Omega \\
\theta(x, t)=\theta_{0}(x, t), \quad(x, t) \in \Omega \times(-\infty, 0]
\end{array}\right.
$$

where $\Omega$ is a bounded domain of $\mathbb{R}^{N}(N \geq 1)$ with a smooth boundary $\partial \Omega, 0 \leq \omega<1$, and $v>0$. The function $g$, external force $f$ and memory kernel $k$ will be specified later.

It is well known that temperature gradients in a plate will contribute to plate deformation. Problem (1.1)-(1.3) can be used to describe the deformation and the temperature distribution of a homogeneous, isotropic and thermoelastic thin material with memory, see $[1,2]$ for the details. Functions $u(x, t)$ and $\theta(x, t)$ represent the displacement and temperature variation field relative to the equilibrium reference value, respectively. The cases

(c) The Author(s) 2018. This article is distributed under the terms of the Creative Commons Attribution 4.0 International License (http://creativecommons.org/licenses/by/4.0/), which permits unrestricted use, distribution, and reproduction in any medium, provided you give appropriate credit to the original author(s) and the source, provide a link to the Creative Commons license, and indicate if changes were made. 
$\omega=0$ and $0<\omega<1$ in $(1.1)_{2}$ are usually referred to as the Gurtin-Pipkin model [3] and the Coleman-Gurtin model [4], respectively. In the absence of thermal effects, (1.1) reduces to an extensible plate equation. This class of equations model the vibrations of extensible elastic beams (when $N=1$ ) and plates (when $N=2$ ), and have been extensively investigated (see, e.g., [5-11] and the references therein).

Wu [12] studied

$$
\left\{\begin{array}{l}
u_{t t}+\Delta^{2} u-\Delta u_{t}+\Delta \theta+f(u)=0, \quad(x, t) \in \Omega \times \mathbb{R}^{+}, \\
\theta_{t}-\int_{0}^{\infty} k(\tau) \Delta \theta(t-\tau) \mathrm{d} \tau-\Delta u_{t}=0, \quad(x, t) \in \Omega \times \mathbb{R}^{+},
\end{array}\right.
$$

subject to (1.2) and (1.3). Under the assumption $f \in C^{2}$, the author obtained the global existence and uniqueness of solutions, as well as the existence of global attractors. Moreover, when $f$ is assumed real analytic, the convergence of global solutions to a single steady state, as time goes to infinity, was proved and also an estimate of the convergence rate was provided. Barbosa and Ma [13] investigated problem (1.1)-(1.3) by adding an extra external force $h$ to $(1.1)_{1}$. Using the assumptions $g, f \in C^{1}$, the authors derived the global well-posedness of solutions, the existence of global attractors with finite fractal dimension, and the existence of exponential attractors.

In the present paper, our purpose is to tackle the global existence of solutions to problem (1.1)-(1.3) under weaker assumptions on $g$ and $f$. As in [12,13], we employ the past history approach $[14,15]$, so that problem (1.1)-(1.3) can be transformed into an equivalent system in the history phase space. By means of the potential well theory [16, 17], we establish the theorems on global existence of solutions by discussing the level of initial energy.

This paper is organized as follows. In Sect. 2, some assumptions on $g, f$ and $k$ are displayed. Moreover, problem (1.1)-(1.3) is transformed into an equivalent system, and the main results of this paper are stated. In Sect. 3, the global existence of solutions with subcritical initial energy is established. In Sect. 4, the global existence of solutions with critical initial energy is derived.

\section{Preliminaries and main results}

\subsection{Notations and assumptions}

Throughout the paper, for simplicity, we denote

$$
\|\cdot\|_{p}:=\|\cdot\|_{L^{p}(\Omega)}, \quad\|\cdot\|:=\|\cdot\|_{2} .
$$

Moreover, $(\cdot, \cdot)$ denotes either the $L^{2}$-inner product or a duality pairing between a space and its dual space.

We make the following assumptions on $g, f$ and $k$, respectively.

( $\left.\mathrm{A}_{1}\right) g \in C(\mathbb{R}), g(z)>0$, and there exists a constant $\alpha>0$ such that $\alpha G(z) \geq z g(z)$, where

$$
G(z)=\int_{0}^{z} g(s) \mathrm{d} s
$$

$\left(\mathrm{A}_{2}\right) f \in C(\mathbb{R})$. There exists a constant $\beta>0$ such that $|f(u)| \leq \beta|u|^{p-1}$, where

$$
2 \leq p<\infty \quad \text { if } N \leq 4, \quad 2 \leq p<\frac{2 N}{N-4} \quad \text { if } N>4 .
$$


Moreover, there exists a constant $\gamma>2 \tilde{\alpha}$ such that $u f(u) \geq \gamma F(u)$, where $\tilde{\alpha}:=$ $\max \{1, \alpha\}$ and

$$
F(u)=\int_{0}^{u} f(s) \mathrm{d} s
$$

(A $\left.\mathrm{A}_{3}\right) k \in C^{2}\left(\mathbb{R}^{+}\right), k(\tau) \geq 0, k^{\prime}(\tau) \leq 0$ and $k^{\prime \prime}(\tau) \geq 0$ for all $\tau \in \mathbb{R}^{+}$. In addition, $\mu(\tau):=$ $-(1-\omega) k^{\prime}(\tau)$.

\subsection{Reformulation of the problem}

We define the auxiliary variable

$$
\psi^{t}(x, \tau)=\int_{0}^{\tau} \theta(x, t-s) \mathrm{d} s, \quad(x, \tau) \in \Omega \times \mathbb{R}^{+}, t \geq 0 .
$$

Thus

$$
-(1-\omega) \int_{0}^{\infty} k(\tau) \Delta \theta(t-\tau) \mathrm{d} \tau=-\int_{0}^{\infty} \mu(\tau) \Delta \psi^{t}(\tau) \mathrm{d} \tau
$$

Consequently, in view of [15, p. 165], problem (1.1)-(1.3) is transformed into the following equivalent system:

$$
\left\{\begin{array}{l}
u_{t t}+\Delta^{2} u-g\left(\|\nabla u\|^{2}\right) \Delta u-\Delta u_{t t}+v \Delta \theta=f(u), \quad(x, t) \in \Omega \times \mathbb{R}^{+}, \\
\theta_{t}-\omega \Delta \theta-\int_{0}^{\infty} \mu(\tau) \Delta \psi^{t}(\tau) \mathrm{d} \tau-v \Delta u_{t}=0, \quad(x, t) \in \Omega \times \mathbb{R}^{+}, \\
\psi_{t}^{t}(x, \tau)=\theta(x, t)-\psi_{\tau}^{t}(x, \tau), \quad(x, \tau) \in \Omega \times \mathbb{R}^{+}, t>0,
\end{array}\right.
$$

with boundary conditions

$$
\left\{\begin{array}{l}
u=\Delta u=0, \quad(x, t) \in \partial \Omega \times[0, \infty), \\
\theta=0, \quad(x, t) \in \partial \Omega \times[0, \infty), \\
\psi^{t}(x, \tau)=0, \quad(x, \tau) \in \partial \Omega \times \mathbb{R}^{+}, t \geq 0,
\end{array}\right.
$$

and initial conditions

$$
\left\{\begin{array}{l}
u(x, 0)=u_{0}(x), \quad u_{t}(x, 0)=u_{1}(x) \\
\theta(x, 0)=\theta_{0}(x) \\
\psi^{0}(x, \tau)=\psi_{0}(x, \tau)
\end{array}\right.
$$

where

$$
\begin{aligned}
& \theta_{0}(x)=\theta_{0}(x, 0), \quad x \in \Omega, \\
& \psi_{0}(x, \tau)=\int_{0}^{\tau} \theta(x,-s) \mathrm{d} s, \quad(x, \tau) \in \Omega \times \mathbb{R}^{+} .
\end{aligned}
$$




\subsection{Statement of main results}

We introduce a weighted $L^{2}$-space

$$
\mathcal{L}:=L_{\mu}^{2}\left(\mathbb{R}^{+} ; H_{0}^{1}(\Omega)\right)=\left\{\psi: \mathbb{R}^{+} \rightarrow H_{0}^{1}(\Omega) \mid \int_{0}^{\infty} \mu(\tau)\|\nabla \psi(\tau)\|^{2} \mathrm{~d} \tau<\infty\right\},
$$

which is a Hilbert space equipped with the inner product

$$
(\psi, \varphi)_{\mathcal{L}}=\int_{0}^{\infty} \mu(\tau)(\nabla \psi, \nabla \varphi) \mathrm{d} \tau
$$

and the norm

$$
\|\psi\|_{\mathcal{L}}^{2}=\int_{0}^{\infty} \mu(\tau)\|\nabla \psi\|^{2} \mathrm{~d} \tau
$$

Definition 2.1 $\left(u(t), \theta(t), v^{t}\right)$ is called a weak solution to problem (2.1)-(2.3) if $u \in$ $L^{\infty}\left(0, T ; H^{2}(\Omega) \cap H_{0}^{1}(\Omega)\right), u_{t} \in L^{\infty}\left(0, T ; H_{0}^{1}(\Omega)\right), \theta \in L^{\infty}\left(0, T ; L^{2}(\Omega)\right), \psi^{t} \in L^{\infty}(0, T ; \mathcal{L})$, $u(x, 0)=u_{0}(x), u_{t}(x, 0)=u_{1}(x), \theta(x, 0)=\theta_{0}(x), \psi^{0}(x, \tau)=\psi_{0}(x, \tau)$, and

$$
\begin{aligned}
& \left(u_{t t}, \varphi_{1}\right)+\left(\Delta u, \Delta \varphi_{1}\right)+\left(g\left(\|\nabla u\|^{2}\right) \nabla u, \nabla \varphi_{1}\right)+\left(\nabla u_{t t}, \nabla \varphi_{1}\right)-v\left(\nabla \theta, \nabla \varphi_{1}\right)=\left(f(u), \varphi_{1}\right), \\
& \left(\theta_{t}, \varphi_{2}\right)+\omega\left(\nabla \theta, \nabla \varphi_{2}\right)+\left(\psi^{t}, \varphi_{2}\right)_{\mathcal{L}}+v\left(\nabla u_{t}, \nabla \varphi_{2}\right)=0, \\
& \left(\psi_{t}^{t}, \varphi_{3}\right)_{\mathcal{L}}=\left(\theta, \varphi_{3}\right)_{\mathcal{L}}-\left(\psi_{\tau}^{t}, \varphi_{3}\right)_{\mathcal{L}},
\end{aligned}
$$

for any $\varphi_{1} \in H^{2}(\Omega) \cap H_{0}^{1}(\Omega), \varphi_{2} \in H_{0}^{1}(\Omega), \varphi_{3} \in \mathcal{L}$ and a.e. $t \in(0, T]$.

The energy associated with problem (2.1)-(2.3) is given by

$$
\begin{aligned}
E(t)= & \frac{1}{2}\left\|u_{t}\right\|^{2}+\frac{1}{2}\left\|\nabla u_{t}\right\|^{2}+\frac{1}{2}\|\Delta u\|^{2}+\frac{1}{2} G\left(\|\nabla u\|^{2}\right) \\
& +\frac{1}{2}\|\theta\|^{2}+\frac{1}{2}\left\|\psi^{t}\right\|_{\mathcal{L}}^{2}-\int_{\Omega} F(u) \mathrm{d} x .
\end{aligned}
$$

Furthermore, we define the energy functional

$$
J\left(u, \theta, \psi^{t}\right)=\frac{1}{2}\|\Delta u\|^{2}+\frac{1}{2} G\left(\|\nabla u\|^{2}\right)+\frac{1}{2}\|\theta\|^{2}+\frac{1}{2}\left\|\psi^{t}\right\|_{\mathcal{L}}^{2}-\int_{\Omega} F(u) \mathrm{d} x,
$$

and the Nehari functional

$$
I\left(u, \theta, \psi^{t}\right)=\|\Delta u\|^{2}+g\left(\|\nabla u\|^{2}\right)\|\nabla u\|^{2}+\|\theta\|^{2}+\left\|\psi^{t}\right\|_{\mathcal{L}}^{2}-\int_{\Omega} u f(u) \mathrm{d} x .
$$

Thus, all nontrivial stationary solutions belong to the Nehari manifold defined by

$$
\mathcal{N}=\left\{\left(u, \theta, \psi^{t}\right) \in \mathcal{H} \backslash\{(0,0,0)\} \mid I\left(u, \theta, \psi^{t}\right)=0\right\},
$$

where $\mathcal{H}:=H^{2}(\Omega) \cap H_{0}^{1}(\Omega) \times L^{2}(\Omega) \times \mathcal{L}$. Then the mountain pass level of $J$ can be characterized as

$$
d=\inf _{\left(u, \theta, \psi^{t}\right) \in \mathcal{N}} J\left(u, \theta, \psi^{t}\right)
$$


We introduce the potential well

$$
\mathcal{W}=\left\{\left(u, \theta, \psi^{t}\right) \in \mathcal{H} \mid I\left(u, \theta, \psi^{t}\right)>0, J\left(u, \theta, \psi^{t}\right)<d\right\} \cup\{(0,0,0)\},
$$

and let

$$
\overline{\mathcal{W}}:=\mathcal{W} \cup \partial \mathcal{W}=\left\{\left(u, \theta, \psi^{t}\right) \in \mathcal{H} \mid I\left(u, \theta, \psi^{t}\right) \geq 0, J\left(u, \theta, \psi^{t}\right) \leq d\right\}
$$

The main results of this paper are stated as follows.

Theorem 2.1 Let assumptions $\left(\mathrm{A}_{1}\right)-\left(\mathrm{A}_{3}\right)$ be fulfilled, $u_{0} \in H^{2}(\Omega) \cap H_{0}^{1}(\Omega), u_{1} \in H_{0}^{1}(\Omega)$, $\theta_{0} \in L^{2}(\Omega), \psi_{0} \in \mathcal{L}$. Assume that $0<E(0)<d$, and $I\left(u_{0}, \theta_{0}, \psi_{0}\right)>0$ or $\left(u_{0}, \theta_{0}, \psi_{0}\right)=(0,0,0)$. Then problem (2.1)-(2.3) admits a global solution $\left(u, \theta, \psi^{t}\right) \in \mathcal{W}$. Moreover,

$$
E(t)+\omega \int_{0}^{t}\|\nabla \theta\|^{2} \mathrm{~d} \tau \leq E(0)
$$

Theorem 2.2 Let assumptions $\left(\mathrm{A}_{1}\right)-\left(\mathrm{A}_{3}\right)$ be fulfilled, $u_{0} \in H^{2}(\Omega) \cap H_{0}^{1}(\Omega), u_{1} \in H_{0}^{1}(\Omega)$, $\theta_{0} \in L^{2}(\Omega), \psi_{0} \in \mathcal{L}$. Assume that $E(0)=d$ and $I\left(u_{0}, \theta_{0}, \psi_{0}\right) \geq 0$. Then problem $(2.1)-(2.3)$ admits a global solution $\left(u, \theta, \psi^{t}\right) \in \overline{\mathcal{W}}$.

\section{Proof of Theorem 2.1}

Let $\left\{w_{j}\right\}_{j=1}^{\infty}$ be an orthogonal basis of $H^{2}(\Omega) \cap H_{0}^{1}(\Omega)$ and let an orthonormal basis of $L^{2}(\Omega)$ be given by eigenfunctions of

$$
\left\{\begin{array}{l}
\Delta^{2} w=\lambda w, \quad \text { in } \Omega, \\
w=\Delta w=0, \quad \text { on } \partial \Omega .
\end{array}\right.
$$

Then $\left\{v_{j}\right\}_{j=1}^{\infty}$ is an orthonormal basis of $H_{0}^{1}(\Omega)$, where $v_{j}=\frac{w_{j}}{\lambda_{j}^{\frac{1}{4}}}$. We select $\left\{e_{j}\right\}_{j=1}^{\infty}$ as $\left\{l_{k} v_{j}\right\}_{k, j=1}^{\infty}$, where $\left\{l_{k}\right\}_{k=1}^{\infty}$ is an orthonormal basis of $L_{\mu}^{2}\left(\mathbb{R}^{+}\right)$. Then $\left\{e_{j}\right\}_{j=1}^{\infty}$ is an orthonormal basis of $\mathcal{L}$. We construct the approximate solutions to problem (2.1)-(2.3) as

$$
\begin{aligned}
& u_{n}(x, t)=\sum_{j=1}^{n} \xi_{j n}(t) w_{j}(x), \quad \theta_{n}(x, t)=\sum_{j=1}^{n} \eta_{j n}(t) v_{j}(x), \\
& \psi_{n}^{t}(x, \tau)=\sum_{j=1}^{n} \zeta_{j n}(t) e_{j}(x, \tau), \quad n=1,2, \ldots
\end{aligned}
$$

which satisfy

$$
\left\{\begin{array}{l}
\left(u_{n t t}, w_{j}\right)+\left(\Delta u_{n}, \Delta w_{j}\right)+\left(g\left(\left\|\nabla u_{n}\right\|^{2}\right) \nabla u_{n}, \nabla w_{j}\right) \\
\quad+\left(\nabla u_{n t t}, \nabla w_{j}\right)-v\left(\nabla \theta_{n}, \nabla w_{j}\right)=\left(f\left(u_{n}\right), w_{j}\right), \\
\left(\theta_{n t}, v_{j}\right)+\omega\left(\nabla \theta_{n}, \nabla v_{j}\right)+\left(\psi_{n}^{t}, v_{j}\right)_{\mathcal{L}}+v\left(\nabla u_{n t}, \nabla v_{j}\right)=0, \\
\left(\psi_{n t}^{t}, e_{j}\right)_{\mathcal{L}}=\left(\theta_{n}, e_{j}\right)_{\mathcal{L}}-\left(\psi_{n \tau}^{t}, e_{j}\right)_{\mathcal{L}}, \quad j=1,2, \ldots, n,
\end{array}\right.
$$


with

$$
\left\{\begin{array}{l}
u_{n}(x, 0)=\sum_{j=1}^{n} \xi_{j n}(0) w_{j}(x) \rightarrow u_{0}(x) \quad \text { in } H^{2}(\Omega) \cap H_{0}^{1}(\Omega) \\
u_{n t}(x, 0)=\sum_{j=1}^{n} \xi_{j n}^{\prime}(0) w_{j}(x) \rightarrow u_{1}(x) \quad \text { in } H_{0}^{1}(\Omega) \\
\theta_{n}(x, 0)=\sum_{j=1}^{n} \eta_{j n}(0) v_{j}(x) \rightarrow \theta_{0}(x) \quad \text { in } L^{2}(\Omega) \\
\psi_{n}^{0}(x, \tau)=\sum_{j=1}^{n} \zeta_{j n}(0) e_{j}(x, \tau) \rightarrow \psi_{0}(x, \tau) \quad \text { in } \mathcal{L} .
\end{array}\right.
$$

The approximate problem (3.1)-(3.2) can be reduced to an ordinary differential system in the variables $\xi_{j n}(t), \eta_{j n}(t)$ and $\zeta_{j n}(t)$. In terms of standard theory for ODEs, there exists a solution $\left(u_{n}(t), \theta_{n}(t), \psi_{n}^{t}\right)$ on some interval $\left[0, T_{n}\right)$ with $T_{n} \leq T$. The following estimates will allow us to extend the local solutions to $[0, T]$ for all $T>0$.

Multiplying $(3.1)_{1}$ by $\xi_{j n}^{\prime}(t),(3.1)_{2}$ by $\eta_{j n}(t)$, and $(3.1)_{3}$ by $\zeta_{j n}(t)$, summing over $j$, and adding the two results, we obtain

$$
\frac{\mathrm{d}}{\mathrm{d} t} E_{n}(t)+\omega\left\|\nabla \theta_{n}\right\|^{2}=-\left(\psi_{n \tau}^{t}, \psi_{n}^{t}\right)_{\mathcal{L}^{\prime}}
$$

where

$$
\begin{aligned}
E_{n}(t)= & \frac{1}{2}\left\|u_{n t}\right\|^{2}+\frac{1}{2}\left\|\nabla u_{n t}\right\|^{2}+\frac{1}{2}\left\|\Delta u_{n}\right\|^{2}+\frac{1}{2} G\left(\left\|\nabla u_{n}\right\|^{2}\right) \\
& +\frac{1}{2}\left\|\theta_{n}\right\|^{2}+\frac{1}{2}\left\|\psi_{n}^{t}\right\|_{\mathcal{L}}^{2}-\int_{\Omega} F\left(u_{n}\right) \mathrm{d} x
\end{aligned}
$$

Since $\psi_{n}^{t}(x, 0)=0$, we deduce from $\left(\mathrm{A}_{3}\right)$ that

$$
\begin{aligned}
\left(\psi_{n \tau}^{t}, \psi_{n}^{t}\right)_{\mathcal{L}} & =\frac{1}{2} \int_{0}^{\infty} \frac{\partial}{\partial \tau}\left(\mu(\tau)\left\|\nabla \psi_{n}^{t}(\tau)\right\|^{2}\right) \mathrm{d} \tau-\frac{1}{2} \int_{0}^{\infty} \mu^{\prime}(\tau)\left\|\nabla \psi_{n}^{t}(\tau)\right\|^{2} \mathrm{~d} \tau \\
& \geq 0
\end{aligned}
$$

Hence, by integrating (3.3) with respect to $t$ from 0 to $t$, we get

$$
E_{n}(t)+\omega \int_{0}^{t}\left\|\nabla \theta_{n}\right\|^{2} \mathrm{~d} \tau \leq E_{n}(0) .
$$

We now claim that

$$
\left(u_{n}(t), \theta_{n}(t), \psi_{n}^{t}\right) \in \mathcal{W},
$$

for all $t \in[0, T]$ and sufficiently large $n$.

Indeed, if $\left(u_{0}, \theta_{0}, \psi_{0}\right)=(0,0,0)$, then $\left(u_{0}, \theta_{0}, \psi_{0}\right) \in \mathcal{W}$. If $I\left(u_{0}, \theta_{0}, \psi_{0}\right)>0$, then, from $E(0)<$ $d$, i.e.,

$$
\frac{1}{2}\left\|u_{1}\right\|^{2}+\frac{1}{2}\left\|\nabla u_{1}\right\|^{2}+J\left(u_{0}, \theta_{0}, \psi_{0}\right)<d
$$

it follows that $J\left(u_{0}, \theta_{0}, \psi_{0}\right)<d$. Hence $\left(u_{0}, \theta_{0}, \psi_{0}\right) \in \mathcal{W}$. Thus $\left(u_{n}(0), \theta_{n}(0), \psi_{n}^{0}\right) \in \mathcal{W}$ for sufficiently large $n$ due to (3.2). As a result, assertion (3.6) follows as desired. If it was not the case, there would exist a $0<t_{0}<T$ such that $\left(u_{n}\left(t_{0}\right), \theta_{n}\left(t_{0}\right), \psi_{n}^{t_{0}}\right) \in \partial \mathcal{W}$, i.e., 
$I\left(u_{n}\left(t_{0}\right), \theta_{n}\left(t_{0}\right), \psi_{n}^{t_{0}}\right)=0$ and $\left(u_{n}\left(t_{0}\right), \theta_{n}\left(t_{0}\right), \psi_{n}^{t_{0}}\right) \neq(0,0,0)$, or $J\left(u_{n}\left(t_{0}\right), \theta_{n}\left(t_{0}\right), \psi_{n}^{t_{0}}\right)=d$. Due to (3.4), (3.5) and (3.2), we get

$$
\frac{1}{2}\left\|u_{n t}\right\|^{2}+\frac{1}{2}\left\|\nabla u_{n t}\right\|^{2}+J\left(u_{n}, \theta_{n}, \psi_{n}^{t}\right)<d,
$$

for all $t \in[0, T]$ and sufficiently large $n$. This tells us that $J\left(u_{n}\left(t_{0}\right), \theta_{n}\left(t_{0}\right), \psi_{n}^{t_{0}}\right)=d$ is impossible. On the other hand, if $I\left(u_{n}\left(t_{0}\right), \theta_{n}\left(t_{0}\right), \psi_{n}^{t_{0}}\right)=0$ and $\left(u_{n}\left(t_{0}\right), \theta_{n}\left(t_{0}\right), \psi_{n}^{t_{0}}\right) \neq(0,0,0)$, then, by the definition of $d$, we get $J\left(u_{n}\left(t_{0}\right), \theta_{n}\left(t_{0}\right), \psi_{n}^{t_{0}}\right) \geq d$, which contradicts (3.7).

We deduce from $\left(\mathrm{A}_{1}\right)$ and $\left(\mathrm{A}_{2}\right)$ that

$$
\begin{aligned}
J\left(u_{n}, \theta_{n}, \psi_{n}^{t}\right) \geq & \frac{\gamma-2 \tilde{\alpha}}{2 \tilde{\alpha} \gamma}\left(\left\|\Delta u_{n}\right\|^{2}+g\left(\left\|\nabla u_{n}\right\|^{2}\right)\left\|\nabla u_{n}\right\|^{2}+\left\|\theta_{n}\right\|^{2}+\left\|\psi_{n}^{t}\right\|_{\mathcal{L}}^{2}\right) \\
& +\frac{1}{\gamma} I\left(u_{n}, \theta_{n}, \psi_{n}^{t}\right) .
\end{aligned}
$$

Combining this with (3.4)-(3.6) and (3.2), we arrive at

$$
\begin{gathered}
\frac{\gamma-2 \tilde{\alpha}}{2 \tilde{\alpha} \gamma}\left(\left\|\Delta u_{n}\right\|^{2}+g\left(\left\|\nabla u_{n}\right\|^{2}\right)\left\|\nabla u_{n}\right\|^{2}+\left\|\theta_{n}\right\|^{2}+\left\|\psi_{n}^{t}\right\|_{\mathcal{L}}^{2}\right) \\
+\frac{1}{2}\left\|u_{n t}\right\|^{2}+\frac{1}{2}\left\|\nabla u_{n t}\right\|^{2}+\omega \int_{0}^{t}\left\|\nabla \theta_{n}\right\|^{2} \mathrm{~d} \tau<d,
\end{gathered}
$$

for all $t \in[0, T]$ and sufficiently large $n$. Moreover,

$$
\left\|f\left(u_{n}\right)\right\|_{q}^{q} \leq \beta^{q}\left\|u_{n}\right\|_{p}^{p} \leq \beta^{q} C_{*}^{p}\left\|\Delta u_{n}\right\|^{p}<\beta^{q} C_{*}^{p}\left(\frac{2 \tilde{\alpha} \gamma}{\gamma-2 \tilde{\alpha}} d\right)^{\frac{p}{2}}
$$

where $q=\frac{p}{p-1}$, and $C_{*}$ is the constant for the Sobolev embedding $H^{2}(\Omega) \cap H_{0}^{1}(\Omega) \hookrightarrow$ $L^{p}(\Omega)$.

Hence there exist $\left(u, \theta, \psi^{t}\right)$ and subsequences of $\left\{u_{n}\right\},\left\{\theta_{n}\right\},\left\{\psi_{n}^{t}\right\}$, still represented by the same notations (and we shall not repeat this), such that, as $n \rightarrow \infty$,

$$
\begin{aligned}
& u_{n} \rightarrow u \quad \text { weakly star in } L^{\infty}\left(0, T ; H^{2}(\Omega) \cap H_{0}^{1}(\Omega)\right), \\
& u_{n} \rightarrow u \quad \text { strongly in } L^{p}(\Omega) \text { and a.e. in } \Omega \times[0, T], \\
& u_{n t} \rightarrow u_{t} \quad \text { weakly star in } L^{\infty}\left(0, T ; H_{0}^{1}(\Omega)\right), \\
& \theta_{n} \rightarrow \theta \quad \text { weakly star in } L^{\infty}\left(0, T ; L^{2}(\Omega)\right) \\
& \quad\left(\text { and weakly in } L^{2}\left(0, T ; H_{0}^{1}(\Omega)\right) \text { if } \omega>0\right), \\
& \psi_{n}^{t} \rightarrow \psi^{t} \quad \text { weakly star in } L^{\infty}(0, T ; \mathcal{L}), \\
& f\left(u_{n}\right) \rightarrow \chi \quad \text { weakly star in } L^{\infty}\left(0, T ; L^{q}(\Omega)\right),
\end{aligned}
$$

for any $T>0$. In view of [18, Lemma 1.3], we have $\chi=f(u)$. According to the Aubin-Lions lemma, we have

$$
u_{n} \rightarrow u \quad \text { strongly in } L^{2}\left(0, T ; H_{0}^{1}(\Omega)\right)
$$


which, together with (3.8), (3.9) and (3.11), gives

$$
u_{n} \rightarrow u \quad \text { strongly in } C\left(0, T ; H_{0}^{1}(\Omega)\right) .
$$

Therefore, by the arguments similar to the proof given in [14, p. 343-345], we can pass to the limit in the approximate problem (3.1)-(3.2). Thus $\left(u, \theta, \psi^{t}\right) \in \mathcal{W}$ is a global solution to problem (2.1)-(2.3).

Next, we prove (2.4). Indeed, note that

$$
\begin{aligned}
\left|G\left(\left\|\nabla u_{n}\right\|^{2}\right)-G\left(\|\nabla u\|^{2}\right)\right| & =\int_{0}^{1} g\left(\vartheta_{1}\right) \mathrm{d} \sigma\left|\left\|\nabla u_{n}\right\|^{2}-\|\nabla u\|^{2}\right| \\
& \leq C_{1}\left\|\nabla u_{n}-\nabla u\right\|,
\end{aligned}
$$

where $\vartheta_{1}=\sigma\left\|\nabla u_{n}\right\|^{2}+(1-\sigma)\|\nabla u\|^{2}, 0<\sigma<1$. Hence it follows from (3.14) that

$$
\lim _{n \rightarrow \infty} G\left(\left\|\nabla u_{n}\right\|^{2}\right)=G\left(\|\nabla u\|^{2}\right) .
$$

Furthermore,

$$
\begin{aligned}
\left|\int_{\Omega} F\left(u_{n}\right) \mathrm{d} x-\int_{\Omega} F(u) \mathrm{d} x\right| & \leq \int_{\Omega}\left|f\left(\vartheta_{2}\right)\right|\left|u_{n}-u\right| \mathrm{d} x \\
& \leq\left\|f\left(\vartheta_{2}\right)\right\|_{q}\left\|u_{n}-u\right\|_{p} \\
& \leq C_{2}\left\|u_{n}-u\right\|_{p},
\end{aligned}
$$

where $\vartheta_{2}=\sigma u_{n}+(1-\sigma) u$. This, together with (3.10), yields

$$
\lim _{n \rightarrow \infty} \int_{\Omega} F\left(u_{n}\right) \mathrm{d} x=\int_{\Omega} F(u) \mathrm{d} x .
$$

Consequently, by (3.9), (3.11)-(3.13), (3.5), (3.15), (3.16) and (3.2), we obtain

$$
\begin{aligned}
\frac{1}{2}\left\|u_{t}\right\|^{2}+\frac{1}{2}\left\|\nabla u_{t}\right\|^{2}+\frac{1}{2}\|\Delta u\|^{2}+\frac{1}{2}\|\theta\|^{2}+\frac{1}{2}\left\|\psi^{t}\right\|_{\mathcal{L}}^{2}+\omega \int_{0}^{t}\|\nabla \theta\|^{2} \mathrm{~d} \tau \\
\leq \liminf _{n \rightarrow \infty}\left(\frac{1}{2}\left\|u_{n t}\right\|^{2}+\frac{1}{2}\left\|\nabla u_{n t}\right\|^{2}+\frac{1}{2}\left\|\Delta u_{n}\right\|^{2}+\frac{1}{2}\left\|\theta_{n}\right\|^{2}+\frac{1}{2}\left\|\psi_{n}^{t}\right\|_{\mathcal{L}}^{2}\right. \\
\left.\quad+\omega \int_{0}^{t}\left\|\nabla \theta_{n}\right\|^{2} \mathrm{~d} \tau\right) \\
\leq \liminf _{n \rightarrow \infty}\left(E_{n}(0)-\frac{1}{2} G\left(\left\|\nabla u_{n}\right\|^{2}\right)+\int_{\Omega} F\left(u_{n}\right) \mathrm{d} x\right) \\
=E(0)-\frac{1}{2} G\left(\|\nabla u\|^{2}\right)+\int_{\Omega} F(u) \mathrm{d} x .
\end{aligned}
$$

Thus the proof of Theorem 2.1 is complete.

\section{Proof of Theorem 2.2}

We divide the proof of this theorem into two cases. 
Case 1. $\left(u_{0}, \theta_{0}, \psi_{0}\right) \neq(0,0,0)$.

Let $\delta_{m}=1-\frac{1}{m}, u_{m 0}=\delta_{m} u_{0}, \theta_{m 0}=\delta_{m} \theta_{0}$ and $\psi_{m 0}=\delta_{m} \psi_{0}, m=2,3, \ldots$. We consider problem (2.1)-(2.2) with the following initial conditions:

$$
\left\{\begin{array}{l}
u(x, 0)=u_{m 0}(x), \quad u_{t}(x, 0)=u_{1}(x) \\
\theta(x, 0)=\theta_{m 0}(x) \\
\psi^{0}(x, \tau)=\psi_{m 0}(x, \tau)
\end{array}\right.
$$

From $I\left(u_{0}, \theta_{0}, \psi_{0}\right) \geq 0$,

$$
\begin{aligned}
J\left(\delta u, \delta \theta, \delta \psi^{t}\right)= & \frac{1}{2} \delta^{2}\|\Delta u\|^{2}+\frac{1}{2} G\left(\delta^{2}\|\nabla u\|^{2}\right)+\frac{1}{2} \delta^{2}\|\theta\|^{2} \\
& +\frac{1}{2} \delta^{2}\left\|\psi^{t}\right\|_{\mathcal{L}}^{2}-\int_{\Omega} F(\delta u) \mathrm{d} x,
\end{aligned}
$$

and

$$
I\left(\delta u, \delta \theta, \delta \psi^{t}\right)=\delta \frac{\mathrm{d}}{\mathrm{d} \delta} J\left(\delta u, \delta \theta, \delta \psi^{t}\right)
$$

it is easy to verify that there exists a unique $\delta_{*}=\delta_{*}\left(u_{0}\right) \geq 1$ such that $J\left(\delta u, \delta \theta, \delta \psi^{t}\right)$ is strictly increasing for $\delta \in\left[0, \delta_{*}\right]$ and assumes the maximum at $\delta=\delta_{*}$. Hence $J\left(u_{m 0}, \theta_{m 0}, \psi_{m 0}\right)<$ $J\left(u_{0}, \theta_{0}, \psi_{0}\right)$ and $I\left(u_{m 0}, \theta_{m 0}, \psi_{m 0}\right)>0$. Moreover,

$$
\begin{aligned}
J\left(u_{m 0}, \theta_{m 0}, \psi_{m 0}\right) \geq & \frac{\gamma-2 \tilde{\alpha}}{2 \tilde{\alpha} \gamma}\left(\left\|\Delta u_{m 0}\right\|^{2}+g\left(\left\|\nabla u_{m 0}\right\|^{2}\right)\left\|\nabla u_{m 0}\right\|^{2}+\left\|\theta_{m 0}\right\|^{2}\right. \\
& \left.+\left\|\psi_{m 0}^{t}\right\|_{\mathcal{L}}^{2}\right)+\frac{1}{\gamma} I\left(u_{m 0}, \theta_{m 0}, \psi_{m 0}\right)
\end{aligned}
$$

$>0$.

We further obtain

$$
E_{m}(0)=\frac{1}{2}\left\|u_{1}\right\|^{2}+\frac{1}{2}\left\|\nabla u_{1}\right\|^{2}+J\left(u_{m 0}, \theta_{m 0}, \psi_{m 0}\right)>0,
$$

and

$$
E_{m}(0)<\frac{1}{2}\left\|u_{1}\right\|^{2}+\frac{1}{2}\left\|\nabla u_{1}\right\|^{2}+J\left(u_{0}, \theta_{0}, \psi_{0}\right)=E(0)=d
$$

Hence, we conclude from Theorem 2.1 that problem (2.1)-(2.2) and (4.1) admits a global solution $\left(u_{m}(t), \theta_{m}(t), \psi_{m}^{t}\right) \in \mathcal{W}$ satisfying

$$
\left\{\begin{array}{l}
\left(u_{m t t}, \varphi_{1}\right)+\left(\Delta u_{m}, \Delta \varphi_{1}\right)+\left(g\left(\left\|\nabla u_{m}\right\|^{2}\right) \nabla u_{m}, \nabla \varphi_{1}\right) \\
\quad+\left(\nabla u_{m t t}, \nabla \varphi_{1}\right)-v\left(\nabla \theta_{m}, \nabla \varphi_{1}\right)=\left(f\left(u_{m}\right), \varphi_{1}\right), \\
\left(\theta_{m t}, \varphi_{2}\right)+\omega\left(\nabla \theta_{m}, \nabla \varphi_{2}\right)+\left(\psi_{m}^{t}, \varphi_{2}\right)_{\mathcal{L}}+v\left(\nabla u_{m t}, \nabla \varphi_{2}\right)=0, \\
\left(\psi_{m t}^{t}, \varphi_{3}\right)_{\mathcal{L}}=\left(\theta_{m}, \varphi_{3}\right)_{\mathcal{L}}-\left(\psi_{m \tau}^{t}, \varphi_{3}\right)_{\mathcal{L}},
\end{array}\right.
$$


with

$$
\left\{\begin{array}{l}
u_{m}(x, 0)=u_{0}(x), \quad u_{m t}(x, 0)=u_{1}(x), \\
\theta_{m}(x, 0)=\theta_{0}(x), \\
\psi_{m}^{0}(x, \tau)=\psi_{0}(x, \tau)
\end{array}\right.
$$

and

$$
E_{m}(t)+\omega \int_{0}^{t}\left\|\nabla \theta_{m}\right\|^{2} \mathrm{~d} \tau \leq E_{m}(0)
$$

Consequently,

$$
\begin{gathered}
\frac{\gamma-2 \tilde{\alpha}}{2 \tilde{\alpha} \gamma}\left(\left\|\Delta u_{m}\right\|^{2}+g\left(\left\|\nabla u_{m}\right\|^{2}\right)\left\|\nabla u_{m}\right\|^{2}+\left\|\theta_{m}\right\|^{2}+\left\|\psi_{m}^{t}\right\|_{\mathcal{L}}^{2}\right) \\
+\frac{1}{2}\left\|u_{m t}\right\|^{2}+\frac{1}{2}\left\|\nabla u_{m t}\right\|^{2}+\omega \int_{0}^{t}\left\|\nabla \theta_{m}\right\|^{2} \mathrm{~d} \tau<d
\end{gathered}
$$

By the arguments similar to the proof of Theorem 2.1, we see that problem (2.1)-(2.3) admits a global solution $\left(u, \theta, \psi^{t}\right) \in \overline{\mathcal{W}}$.

Case 2. $\left(u_{0}, \theta_{0}, \psi_{0}\right)=(0,0,0)$.

In this case, it is clear that $J\left(u_{0}, \theta_{0}, \psi_{0}\right)=0$. Thus

$$
E(0)=\frac{1}{2}\left\|u_{1}\right\|^{2}+\frac{1}{2}\left\|\nabla u_{1}\right\|^{2}
$$

Let $\delta_{m}=1-\frac{1}{m}$ and $u_{m 1}=\delta_{m} u_{1}(x), m>1$ and consider problem (2.1)-(2.2) with the following initial conditions:

$$
\left\{\begin{array}{l}
u(x, 0)=u_{0}(x), \quad u_{t}(x, 0)=u_{m 1}(x) \\
\theta(x, 0)=\theta_{0}(x) \\
\psi^{0}(x, \tau)=\psi_{0}(x, \tau)
\end{array}\right.
$$

Note that

$$
0<E_{m}(0)=\frac{1}{2}\left\|u_{m 1}\right\|^{2}+\frac{1}{2}\left\|\nabla u_{m 1}\right\|^{2}<E(0) .
$$

We conclude from Theorem 2.1 that problem (2.1)-(2.2) and (4.2) admits a global solution $\left(u_{m}, \theta_{m}, \psi_{m}^{t}\right) \in \mathcal{W}$. The remainder of the proof is the same as in Case 1.

\section{Acknowledgements}

The author would like to thank the reviewers for the valuable suggestions.

Funding

This work is supported by the Science and Technology Plan Project of Gansu Province in China (Grant No. 17JR5RA279).

\section{List of abbreviations}

$\|u\|_{p},\|u\|_{L^{p}(\Omega)} ;\|u\|,\|u\|_{2} ; \mathcal{L}, L_{\mu}^{2}\left(\mathbb{R}^{+} ; H_{0}^{1}(\Omega)\right) ; \mathcal{H}, H^{2}(\Omega) \cap H_{0}^{1}(\Omega) \times L^{2}(\Omega) \times \mathcal{L}$. 
Competing interests

The author declares that he has no competing interests.

\section{Authors' contributions}

The author carried out all the work in this manuscript, and read and approved the final manuscript.

\section{Publisher's Note}

Springer Nature remains neutral with regard to jurisdictional claims in published maps and institutional affiliations.

Received: 2 July 2018 Accepted: 17 October 2018 Published online: 24 October 2018

\section{References}

1. Lagnese, J.E.: Boundary Stabilization of Thin Plates. SIAM, Philadelphia (1989)

2. Lagnese, J.E., Lions, J.L.: Modelling Analysis and Control of Thin Plates. Masson, Paris (1988)

3. Gurtin, M.E., Pipkin, A.C.: A general theory of heat conduction with finite wave speeds. Arch. Ration. Mech. Anal. 31(2), 113-126 (1968)

4. Coleman, B.D., Gurtin, M.E.: Equipresence and constitutive equations for rigid heat conductors. Z. Angew. Math. Phys. 18(2), 199-208 (1967)

5. Ball, J.M.: Stability theory for an extensible beam. J. Differ. Equ. 14(3), 399-418 (1973)

6. Berger, H.M.: A new approach to the analysis of large deflections of plates. J. Appl. Mech. 22, 465-472 (1955)

7. Cavalcanti, M.M., Domingos Cavalcanti, V.N., Soriano, J.A.: Global existence and asymptotic stability for the nonlinear and generalized damped extensible plate equation. Commun. Contemp. Math. 6(5), 705-731 (2004)

8. Dickey, R.W.: Free vibrations and dynamic buckling of the extensible beam. J. Math. Anal. Appl. 29(2), 443-454 (1970)

9. Eden, A., Milani, A.J.: Exponential attractors for extensible beam equations. Nonlinearity 6(3), 457-479 (1993)

10. Ma, T.F., Narciso, V.: Global attractor for a model of extensible beam with nonlinear damping and source terms. Nonlinear Anal. 73(10), 3402-3412 (2010)

11. Woinowsky-Krieger, S.: The effect of an axial force on the vibration of hinged bars. J. Appl. Mech. 17, 35-36 (1950)

12. Wu, H.: Long-time behavior for a nonlinear plate equation with thermal memory. J. Math. Anal. Appl. 348(2), 650-670 (2008)

13. Barbosa, A.R.A., Ma, T.F.: Long-time dynamics of an extensible plate equation with thermal memory. J. Math. Anal. Appl. 416(1), 143-165 (2014)

14. Giorgi, C., Pata, V., Marzocchi, A.: Asymptotic behavior of a semilinear problem in heat conduction with memory. Nonlinear Differ. Equ. Appl. 5(3), 333-354 (1998)

15. Grasselli, M., Pata, V.: Uniform attractors of nonautonomous dynamical systems with memory. In: Lorenzi, A., Ruf, B. (eds.) Evolution Equations, Semigroups and Functional Analysis, 1st edn. vol. 50, pp. 155-178. Birkhäuser, Basel (2002)

16. Payne, L.E., Sattinger, D.H.: Sadle points and instability of nonlinear hyperbolic equations. Isr. J. Math. 22(3-4), 273-303 (1975)

17. Sattinger, D.H.: On global solution of nonlinear hyperbolic equations. Arch. Ration. Mech. Anal. 30(2), 148-172 (1968)

18. Lions, J.L.: Quelques Méthodes de Résolution des Problèmes aux Limites Non Linéaires. Dunod, Paris (1969)

\section{Submit your manuscript to a SpringerOpen ${ }^{\circ}$ journal and benefit from:}

- Convenient online submission

- Rigorous peer review

- Open access: articles freely available online

- High visibility within the field

- Retaining the copyright to your article

Submit your next manuscript at $\boldsymbol{\triangleright}$ springeropen.com 